\title{
Almost exponential decay of Benard convection problem without surface tension
}

\author{
Binqiang Xie ${ }^{1}$ and Lan Zeng ${ }^{2}$ \\ ${ }^{1}$ Institute of Applied Physics and Computational Mathematics \\ ${ }^{2}$ The Graduate School of China Academy of Engineering Physics
}

October 1, 2020

\begin{abstract}
We consider the dynamics of an Boussinesq approximation Benard convection uid evolving in a three-dimensional domain bounded below by a xed atten boundary and above by a free moving surface. The domain is horizontally periodic and the eect of the surface tension is neglected on the free surface. By developing a priori estimates for the model, we prove the global existence and almost exponential decay of solutions in the framework of high regularity.
\end{abstract}

\section{Hosted file}

rb.pdf available at https://authorea.com/users/363496/articles/484248-almost-exponentialdecay-of-benard-convection-problem-without-surface-tension 